\title{
Toxoplasmic Encephalitis with Untreated Hairy Cell Leukemia Variant
}

\author{
Taichi Ikebe ${ }^{1,2}$, Hitohiro Sasaki ${ }^{1,3}$, Hiroyuki Takata ${ }^{2}$, Yasuhiko Miyazaki ${ }^{2}$, \\ Eiichi Ohtsuka ${ }^{2}$, Yoshio Saburi ${ }^{2}$, Masao Ogata ${ }^{3}$ and Kuniaki Shirao ${ }^{3}$
}

\begin{abstract}
Toxoplasmic encephalitis is a rare infectious complication in patients with hematological malignancy except for allogeneic hematopoietic stem cell transplantation (HSCT). We herein report a case of possible toxoplasmic encephalitis with untreated hairy cell leukemia variant. Magnetic resonance imaging showed multiple nodules with surrounding edema in the entire cerebrum. A polymerase chain reaction analysis for Toxoplasma gondii was negative. Her signs and symptoms fully recovered by empirical therapy with sulfadiazine and pyrimethamine. Toxoplasmic encephalitis may occur in patients who undergo non-allogeneic HSCT for hematological malignancies, even in those who have not been treated.
\end{abstract}

Key words: toxoplasmic encephalitis, untreated hematological malignancy, hairy cell leukemia variant, brain biopsy

(Intern Med 55: 3175-3180, 2016)

(DOI: 10.2169/internalmedicine.55.6668)

\section{Introduction}

Toxoplasma is a protozoan parasite that infects up to onethird of the world's population (1). Infection is mainly by ingestion or handing of undercooked or raw meat containing tissue cysts or water or food containing oocysts from the feces of infected cats (1). The prevalence of Toxoplasma infection in the general population varies throughout the world, and high prevalence rates have been found Latin America and tropical African countries. Moderate prevalence rates (30 to 50\%) have been found in countries of Central and Southern Europe (2), whereas the reported seroprevalence of anti-Toxoplasma antibodies among pregnant woman in Japan is $10.3 \%$ (3). Due to this low seroprevalence, the incidence of Toxoplasma infection is not high in Japan.

The majority of acute infections in an immunocompetent host are self-limiting. In an immunocompromised host, however, Toxoplasma may cause life-threatening disease, such as encephalitis, pneumonia, and disseminated infections (1). Patients with acquired immunodeficiency syndrome (AIDS) (4) and hematopoietic stem cell transplanta- tion (HSCT) recipients are at a high risk of severe toxoplasmosis (5-7). Recently, toxoplasmosis has been reported in non-allografted HSCT patients with hematological malignancies, mainly treated with adenosine analogs such as fludarabine or pentostatin (8). However, toxoplasmosis in patients with untreated hematological malignancies is thought to be extremely rare $(9,10)$. We herein report a patient with untreated hairy cell leukemia variant who developed toxoplasmic encephalitis (TE).

\section{Case Reports}

A 74-year-old woman was admitted to our hospital because of lethargy, mental disturbance, and right hemiplegia. She had not eaten raw meat, had no contact with animals, and had not been exposed to cat feces. On the day of admission, the patient was afebrile, with a temperature of $36.9^{\circ} \mathrm{C}$. A physical examination revealed a palpable spleen $12 \mathrm{~cm}$ below the left costal margin. A neurological examination showed a decreased gag reflex, an increased tendon reflex, and numbness of the right upper limb.

A computed tomography (CT) scan revealed multiple

${ }^{1}$ Department of Hematology, Almeida Memorial Hospital, Japan, ${ }^{2}$ Department of Hematology, Oita Prefectural Hospital, Japan and ${ }^{3}$ Department of Medical Oncology and Hematology, Oita University Faculty of Medicine, Japan Received for publication October 8, 2015; Accepted for publication March 21, 2016 Correspondence to Dr. Taichi Ikebe, tikebe@oita-u.ac.jp 


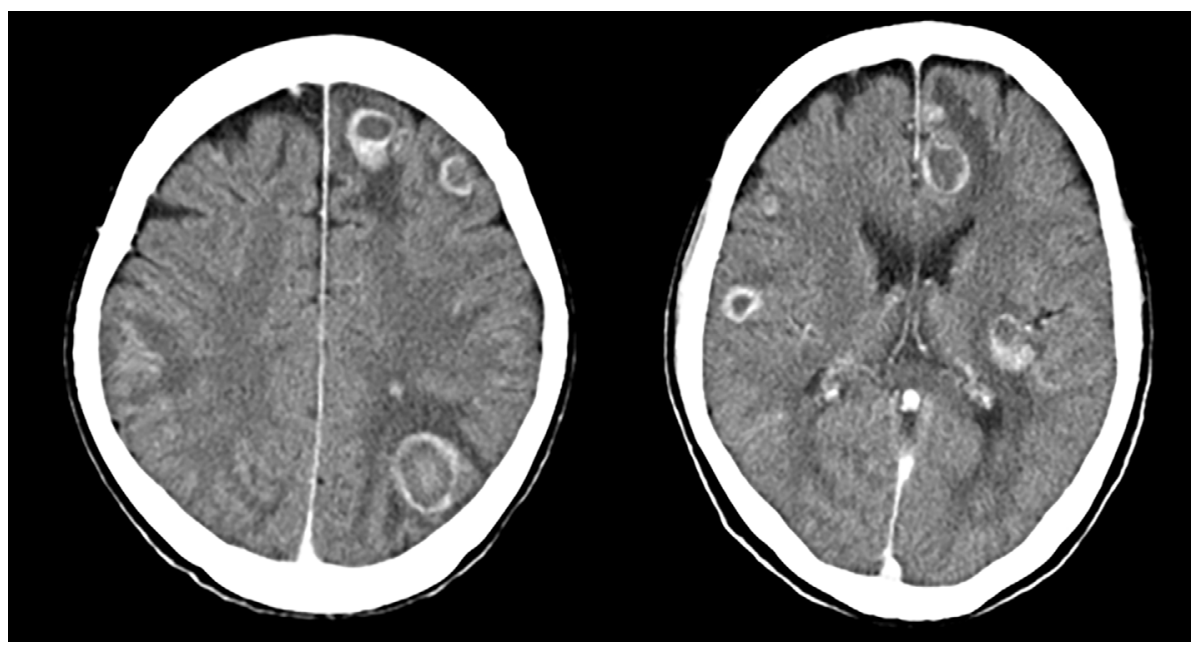

Figure 1. Computed tomography (CT) of the head on admission. Contrast-enhanced CT demonstrates multiple high-density, round, cystic masses with surrounding edema in the entire cerebrum.

ring-enhancing nodules with surrounding edema in the entire cerebrum (Fig. 1). The cerebral spinal fluid (CSF) had a cell count of $3 / \mu \mathrm{L}$. The CSF protein level was $30 \mathrm{mg} / \mathrm{dL}$ with a glucose level of $65 \mathrm{mg} / \mathrm{dL}$ (blood glucose level was $101 \mathrm{mg} /$ $\mathrm{dL})$. The white blood cell count was $48,030 / \mathrm{mm}^{3}$ with $85.5 \%$ abnormal lymphocytes, hemoglobin was $8.5 \mathrm{~g} / \mathrm{dL}$, platelet count was $6.5 \times 10^{4} / \mathrm{mm}^{3}$, and lactate dehydrogenase was $177 \mathrm{IU} / \mathrm{L}$. The CD4-positive T-cell count was $1,561 / \mu \mathrm{L}$. The soluble interleukin-2 receptor level was elevated at 3,530 U/mL. An interferon-gamma assay for tuberculosis was negative. Testing for antibodies against human immunodeficiency virus 1 and human T-lymphotropic virus type 1 yielded negative results. She also tested negative for Toxoplasma-specific immunoglobulin $\mathrm{M}(\operatorname{IgM})$ and positive for immunoglobulin $\mathrm{G}$ ( $\operatorname{IgG})$. A peripheral smear showed increased large abnormal lymphocytes with round nuclei, abundant cytoplasm, and villous-like projections (Fig. 2). A bone marrow aspirate showed weak staining of tartrateresistant acid phosphatase (TRAP) in abnormal lymphocytes (Fig. 2). On flow cytometric analysis, these cells were positive for CD11c, CD19, CD20, CD23, CD103, and lambda light chain, and negative for $\mathrm{CD} 25$. BRAF V600E mutation could not be examined. According to the clinical and laboratory findings, hairy cell leukemia variant (HCL-V) was thus diagnosed.

After admission, the patient exhibited progressive neurological disturbances, including disorientation, short-term memory loss, paralysis of the right upper limb, and drooping of the right corner of the mouth. Magnetic resonance imaging (MRI) of the brain showed multiple nodules in the entire cerebrum with surrounding edema (Fig. 3). According to the clinical signs and symptoms and the imaging findings, TE and infiltration of lymphoma cells were suspected. On funduscopic examination, there was no sign of toxoplasmic chorioretinitis.

Six days after the patient was admitted, we decided to treat her empirically for TE, and combination therapy was started with sulfamethoxazole/trimethoprim and clindamycin.
Nevertheless, her neurological signs gradually worsened, and she developed gait disturbance and aphasia. Eleven days after admission, a cerebral biopsy under local anesthesia was performed. There were foci of necrosis with mild inflammatory change, while no evidence of Toxoplasma gondii zoites or lymphoma cells was detected. Although polymerase chain reaction (PCR) results for T. gondii performed on CSF samples at admission and the cerebral tissue fragments were negative, TE was suspected according to the cranial CT scan and the MRI findings (11). HCL-V infiltration of the central nervous system (CNS) can also be considered the cause of multiple ring-enhancing nodules. Because high-dose methotrexate (HD-MTX) and whole brain radiation therapy can pose a higher risk for persons with older age or in poor condition, anti-Toxoplasma therapy was therefore prioritized.

Twelve days after the patient's admission, the antiToxoplasma therapies were modified. She was started on oral sulfadiazine ( $1 \mathrm{~g}, 4$ times daily) and oral pyrimethamine (50 mg) plus folinic acid (15 mg, once daily), with the discontinuation of sulfamethoxazole/trimethoprim and clindamycin. Sulfadiazine and pyrimethamine were occasionally withdrawn due to nausea and vomiting. Seventeen days after admission, the patient was treated with pentostatin $4 \mathrm{mg} / \mathrm{m}^{2}$ by intravenous injection. Within 1 week, the lymphoma cells in the peripheral blood decreased from about $56,670 / \mathrm{mm}^{3}$ to $790 / \mathrm{mm}^{3}$, and rituximab $375 \mathrm{mg} / \mathrm{m}^{2}$ was then administered.

Although the brain mass was enlarged and the surrounding edema had worsened 1 week after sulfadiazine and pyrimethamine were commenced (Fig. 3), the patient's clinical signs and symptoms gradually improved after 3 weeks on this regimen. After 4 weeks, her gait disturbance and aphasia had completely resolved, without any therapy for CNS lymphoma. Axial MRI of the head showed a marked decrease in the size of the brain nodules, and gadolinium contrast regions disappeared (Fig. 4). After the patient had been on sulfadiazine and pyrimethamine for 12 weeks, longterm prophylactic treatment with sulfamethoxazole and trimethoprim was prescribed. Approximately 1 year after re- 


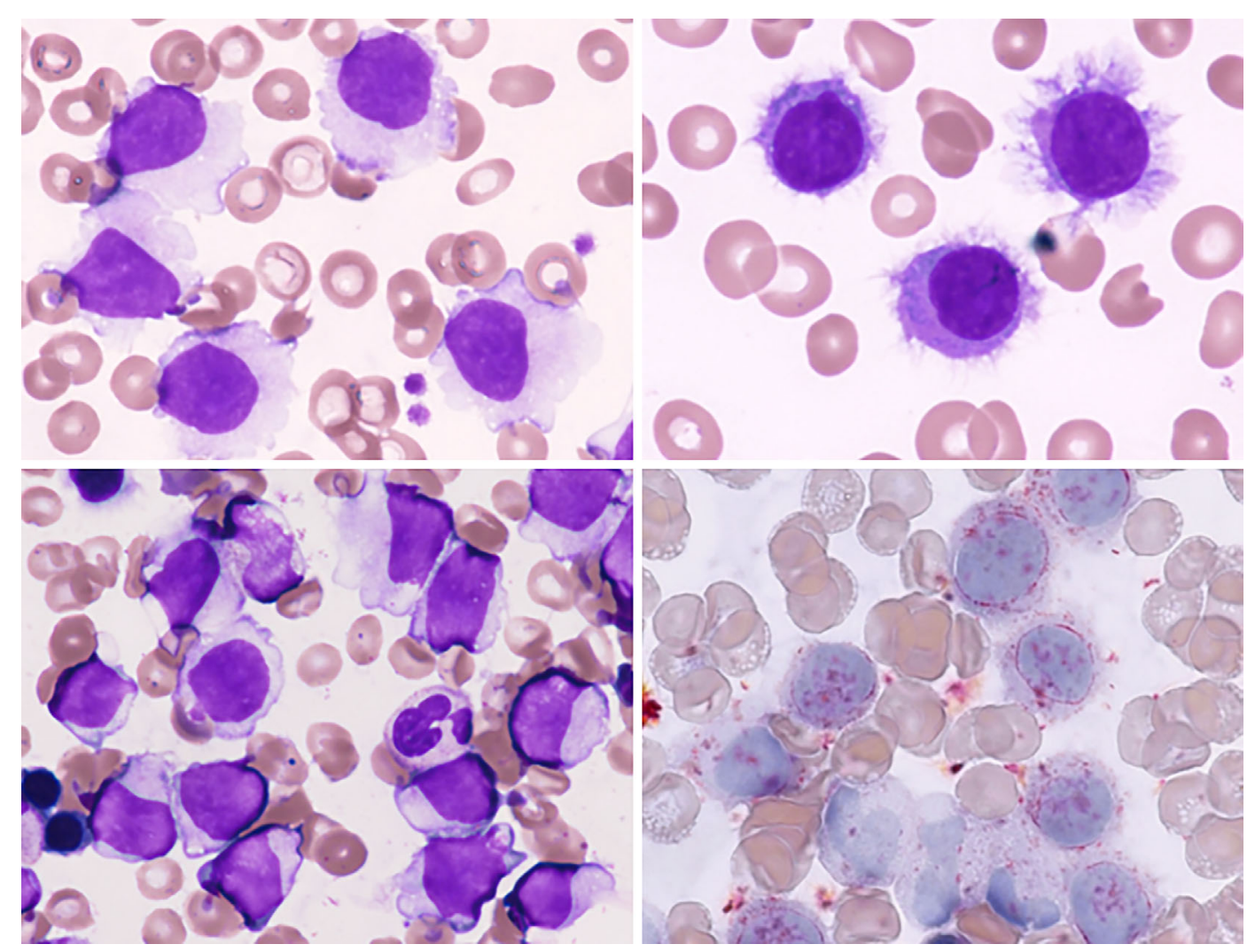

Figure 2. Peripheral smear and bone marrow examination. Circulating large abnormal lymphocytes with round nuclei, abundant cytoplasm (upper left side), and villous-like projections (upper right side) $(400 \times$, Wright-Giemsa stain). Bone marrow aspiration shows increasing large abnormal lymphocyte (lower left side) (400x, Wright-Giemsa stain). TRAP-stain positivity in abnormal lymphocytes is observed (lower right side).

ceiving 8 cycles of rituximab therapy, the patient achieved complete remission without TE recurrence.

\section{Discussion}

Toxoplasmosis is an opportunistic infectious disease in immunocompromised hosts, occurring mainly in patients with AIDS (4). In patients with cancer, the incidence is low. Toxoplasmosis has also been reported in patients undergoing allogeneic stem cell transplantation (5-7). On the other hand, there are a few reports about toxoplasmosis in non-HSCT patients with hematological malignancies (8-10).

A prospective study performed at an institution in India reported that acute toxoplasmosis (defined by positive PCR results for Toxoplasma in the blood or presence of specific $\operatorname{IgM}$ antibody) occurred in 22 of 162 (14\%) non-allogeneic HSCT febrile patients with hematological malignancies (12). These patients presented mainly with respiratory symptoms or organomegaly, and no patient was demonstrated to have a CNS infection. Conversely, in our case the patient did not present with a febrile episode or respiratory symptoms, and she tested negative for Toxoplasma-specific IgM and positive for IgG. TE may have developed from Toxoplasma reactivation of a latent infection.

In a recent report from an institution in France, 2 patients with B-cell lymphoma developed toxoplasmosis, and the incidence of lymphoproliferative diseases was estimated to be $0.006 \%(2 / 3,250)(8)$. Only 2 cases of toxoplasmosis in patients with untreated hematological malignancies were reported. One was a patient with Hodgkin's lymphoma who demonstrated CNS toxoplasmosis diagnosed by antibodies in the serum and CSF (9). In the other case, toxoplasmic myelitis was confirmed by an autopsy of a patient with adult Tcell leukemia/lymphoma (10).

HCL-V accounts for 10-20\% of patients with HCL, and it was categorized as a new entity in the 2008 WHO Classification of Tumours of Haematopoietic and Lymphoid Tissue (13). HCL-V differs from classical HCL (HCL-C) because of the absence of monocytopenia/pancytopenia. Patients with $\mathrm{HCV}-\mathrm{V}$ have an elevated white blood count, easy-to-aspirate bone marrow and negative or weak reactivity to TRAP. Immunophenotypically, HCV-V cells are positive for B-cell antigen, CD103 and CD11c and negative for CD25 (13). Recently, the BRAF V600E mutation, which is a serine/threonine kinase with a regulatory role in the mitogen-activated protein kinase (MAPK) signaling pathway, has been reported to be observed in almost all cases of HCL-C, whereas it is negative in other B-cell malignancies, including HCL-V (14). Unfortunately, we could not examine $B R A F$ V600E mutation in the present patient because this 


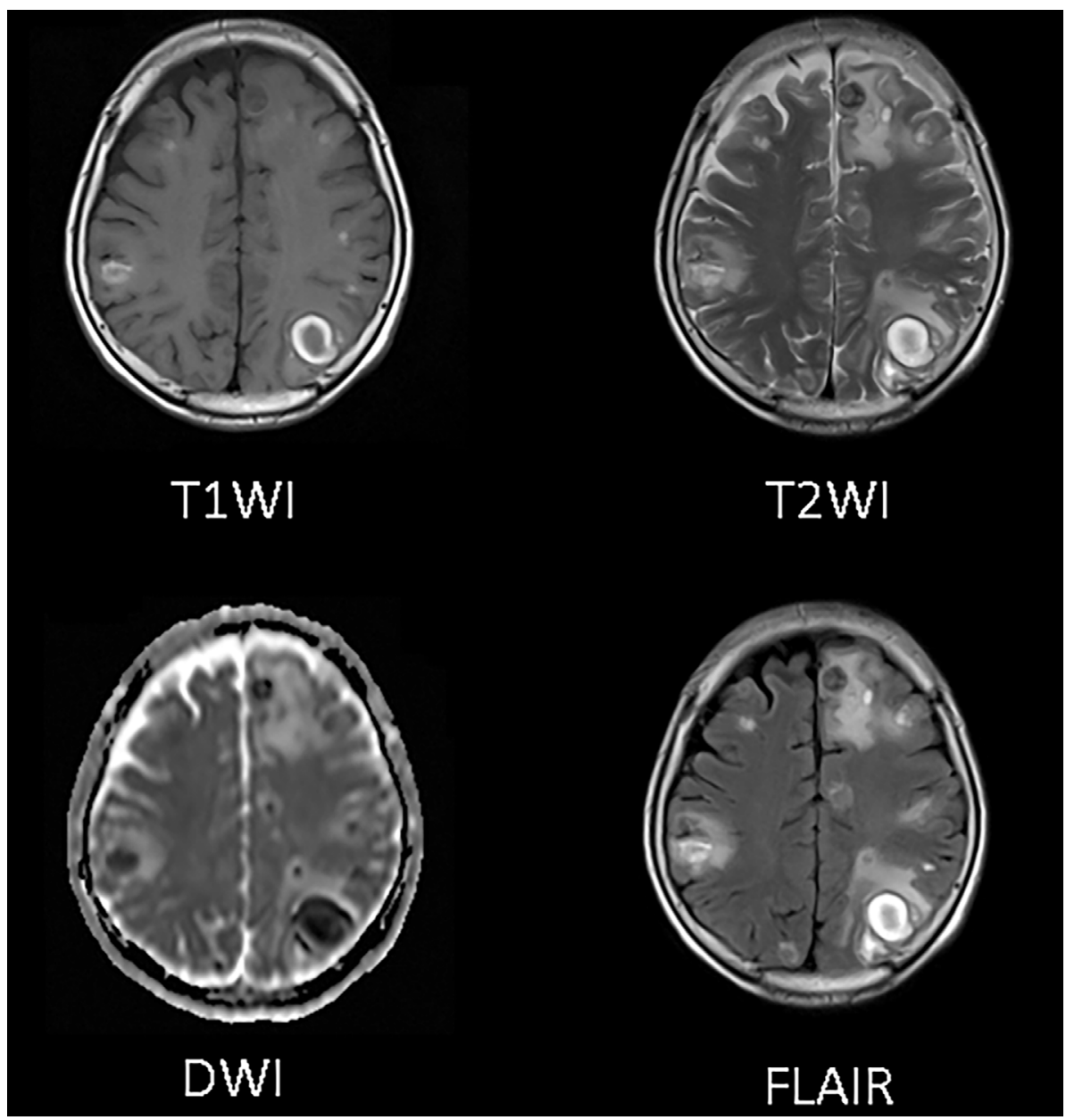

Figure 3. Magnetic resonance images (MRI) of the head prior to initiation of anti-Toxoplasma therapy. The axial T1- and T2-weighted MRI images show high intensity in the marginal lesions and low intensity in the lesions in the cerebrum. In the T2-weighted, diffusion-weighted images and fluid-attenuated inversion recovery images, high-intensity areas are observed around the lesions in the white matter.

mutation cannot be investigated in commercial clinical laboratories. HCL-C also demonstrates underlying immune deficits from monocytopenia, neutropenia, and T-cell/NK-cell impairment, which cause opportunistic infections, including Toxoplasma infection (15). On the other hand, the present patient showed TE with untreated HCL-V. Little has been reported on the inherent immune status of HCL-V. The largest study conducted on HCL-V reported that a frequent cause of death was disease-related, while opportunistic infection was not cited (16). To the best of our knowledge, this is the first report of a patient with TE and untreated HCL-V, which we believe indicates that patients with untreated HCL-V may have immune deficits that cause opportunistic infections.

The most difficult clinical issue is making a definitive diagnosis of the CNS lesions. The differential diagnoses include infiltration of lymphoma cells, metastatic brain tumors, toxoplasmosis, and tuberculosis. In general, CNS lymphoma lesions demonstrate a solitary mass, while about onethird of them have multiple nodules (17). Furthermore, CNS lymphoma is characterized by patchy and uniform enhance- ment on MRI, and occasionally low-density areas reflecting necrosis and bleeding that resemble TE and tuberculosis (18). It is therefore difficult to correctly diagnose CNS lesions. In a previous case report, a patient with AIDS showed multiple masses with enhancement effects seen on CT imaging and no accumulation seen on thallium scintigraphy. Although the enhancement effects improved after the administration of empirical sulfadiazine and pyrimethamine, the mass lesions were not reduced. After the patient had received 4 weeks of empirical therapy, a brain biopsy was performed, and the pathological diagnosis was malignant lymphoma (19).

Although almost all CNS lymphoma cases are of the diffuse large B-cell type, about 10\% consist of low-grade lymphomas, Burkitt lymphomas, or T-cell lymphomas (17). There has been one report on HCL-C infiltration of the CNS (20). However, no cases of HCL-V with CNS involvement have been reported. In the present case, infiltration of lymphoma cells was not confirmed by an immunohistochemical analysis. Chemotherapy regimens incorporating HD-MTX are considered to be the standard care as induc- 


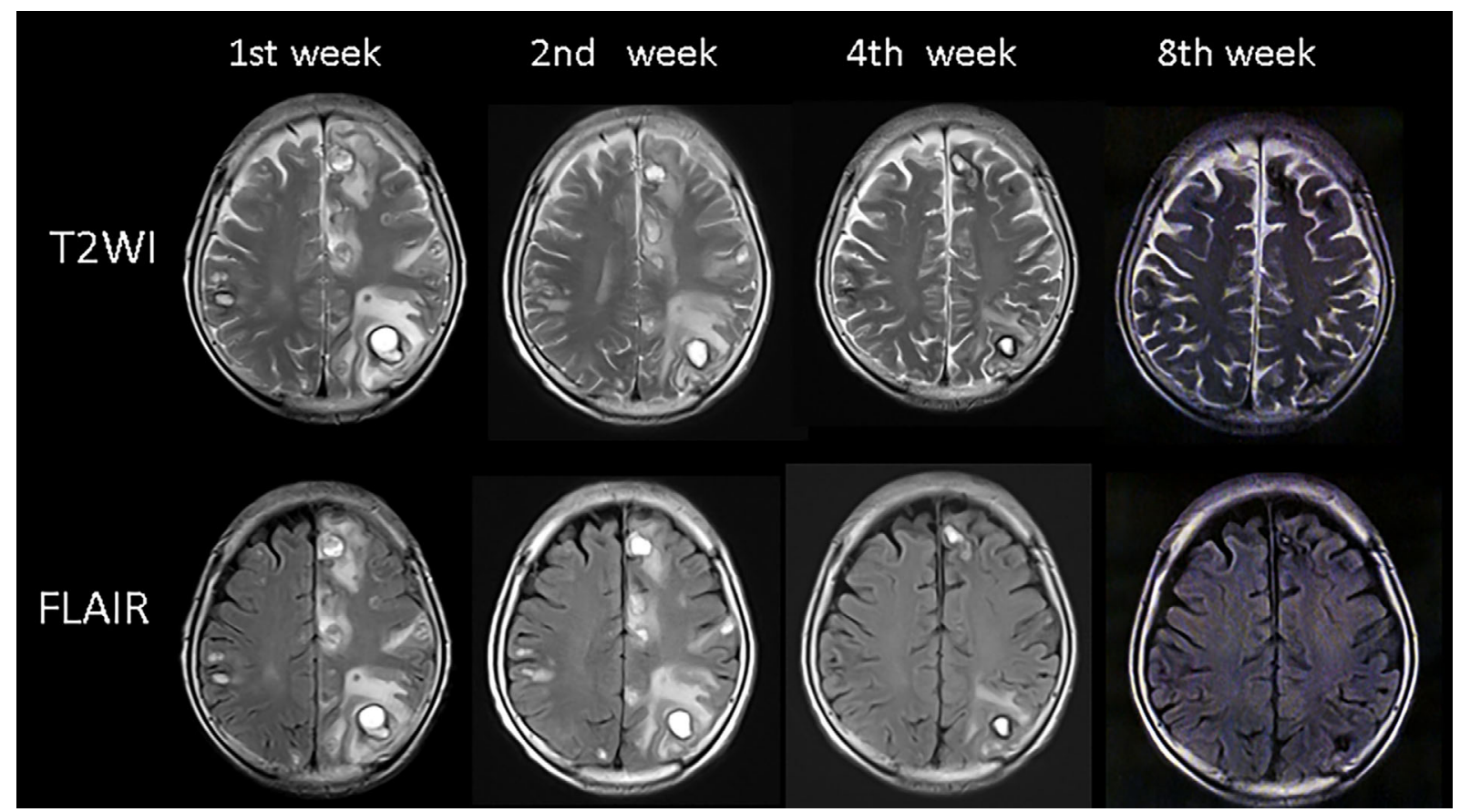

Figure 4. MRI findings after initiation of specific anti-Toxoplasma therapy. The intracranial masses have increased and there is exacerbation of the surrounding edema 1 week after initiation of sulfadiazine and pyrimethamine. These findings are subsequently improved.

tion and salvage therapy for CNS lymphoma because many chemotherapeutic drugs for lymphoma do not penetrate the blood-brain barrier (BBB). In fact, pentostatin and rituximab penetrate the $\mathrm{BBB}$, with concentrations in the CSF reaching only $10-12 \%(21)$ and $0.1-4.4 \%(22)$, respectively. These pharmacodynamics of chemotherapeutic drugs and the clinical course indicate that CNS lymphoma was not the cause of CNS lesions in the present case.

TE is a life-threatening infection in immunocompromised hosts; thus, an early diagnosis and early therapeutic intervention are important (11). Clinical manifestations of TE are often nonspecific with a fever, headache, mental disturbance, focal motor deficits, cranial nerve disturbances, sensory abnormalities, cerebellar signs (1). Therefore, TE must be carefully considered in the differential diagnosis of a wide spectrum of clinical presentations. When clinical signs indicate involvement of the CNS, serological testing for Toxoplasma-specific IgG and neuroimaging studies such as CT or MRI should be performed immediately (1).

A definitive diagnosis of TE is made by a histological examination, isolation of protozoa, or detection of Toxoplasma DNA by PCR $(1,2)$. However, TE is often difficult to diagnose histologically in patients with hematological malignancy due to bleeding tendency, immunodeficiency, and general status. Direct detection of protozoa by tissue culture or mouse inoculation is evidence of infection, but is timeconsuming. Thus, PCR has been widely used for the diagnosis of TE due to convenience and rapidity. Moreover, the PCR technique is applicable for blood, CSF, bronchoalveolar fluid and tissue samples (2).

The problem of using PCR for the diagnosis of TE is the possibility of obtaining false negative results. In TE in an immunocompromised host, Toxoplasma DNA can be detected in the CSF with a high specificity between 96-100\%, however, the sensitivity varies between $33-83 \%(2,23)$. Thus, a positive PCR test result indicates a diagnosis of TE, but a negative result cannot exclude it. Although TE was strongly suspected by clinical manifestation and typical neuroimaging findings in the present case, no evidence of Toxoplasma infection was detected by histological and PCR methods. In the present study, empirical treatment with sulfamethoxazole/trimethoprim and clindamycin initiated before the biopsy may have influenced the pathological findings, however, PCR tests for brain tissue and CSF before starting empirical treatment also revealed negative results. In a previous report of toxoplasmosis in patients after HSCT, Martino et al. proposed definitions for toxoplasmosis (11): definite findings include histologic, cytologic, or culture evidence in tissue samples; probable (PCR-documented) findings include clinical and radiologic evidence of organ involvement plus at least one positive PCR result from the blood, CSF, or bronchial wash, without histologic confirmation; and possible (imaging documented) findings include radiologic evidence of central nervous system toxoplasmosis and response to therapy with no other explanation (11). The present case was diagnosed with possible TE according to these criteria. The same condition may occur in practice, and empiric antiToxoplasma treatment is acceptable for immunocompromised patients with multiple ring-enhancing brain lesions and positive IgG titers against Toxoplasma. In this case, the neurological signs and symptoms had deteriorated rapidly, and empirical therapy with sulfadiazine and pyrimethamine 
was started. This early intervention might have led to the recovery from severe TE.

TE may occur in patients who undergo non-allogeneic HSCT for hematological malignancies, even in those who have not been treated. The early administration of Toxoplasma-specific therapy can lead to a favorable outcome, and empirical therapy should be initiated to avoid a delay in therapy.

The authors state that they have no Conflict of Interest (COI).

\section{Acknowledgement}

The authors are grateful to K. Norose (Department of Infection and Host Defense, Graduate School of Medicine, Chiba University) for examination of $T$. gondii with PCR method, to M. Mawatari and Y. Kato (Disease Control and Prevention Center, National Center for Global Health and Medicine, Tokyo, Japan) for arrangement of sulfadiazine and pyrimethamine.

\section{References}

1. Montoya JG, Liesenfeld O. Toxoplasmosis. Lancet 363: 19651976, 2004.

2. Robert-Gangneux F, Dardé ML. Epidemiology of and diagnostic strategies for toxoplasmosis. Clin Microbiol Rev 25: 264-296, 2012.

3. Sakikawa M, Noda S, Hanaoka M, et al. Anti-Toxoplasma antibody prevalence, primary infection rate, and risk factors in a study of toxoplasmosis in 4,466 pregnant women in Japan. Clin Vaccine Immunol 19: 365-367, 2012.

4. Porter SB, Sande MA. Toxoplasmosis of the central nervous system in the acquired immunodeficiency syndrome. N Engl J Med 327: 1643-1648, 1992.

5. Cibickova L, Horacek J, Prasil P, et al. Cerebral toxoplasmosis in an allogeneic peripheral stem cell transplant recipient: case report and review of literature. Transpl Infect Dis 9: 332-335, 2007.

6. Hakko E, Ozkan HA, Karaman K, Gulbas Z. Analysis of cerebral toxoplasmosis in a series of 170 allogeneic hematopoietic stem cell transplant patients. Transpl Infect Dis 15: 575-580, 2013.

7. Sumi M, Aosai F, Norose $\mathrm{K}$, et al. Acute exacerbation of Toxoplasma gondii infection after hematopoietic stem cell transplantation: five case reports among 279 recipients. Int J Hematol 98: 214-222, 2013.

8. Scerra S, Coignard-Biehler H, Lanternier F, et al. Disseminated toxoplasmosis in non-allografted patients with hematologic malignancies: report of two cases and literature review. Eur J Clin Microbiol Infect Dis 32: 1259-1268, 2013.

9. Green JA, Spruance SL, Cheson BD. Favorable outcome of central nervous system toxoplasmosis occurring in a patient with untreated Hodgkin's disease. Cancer 45: 808-810, 1980.

10. Maciel E, Siqueira I, Queiroz AC, Melo A. Toxoplasma gondii myelitis in a patient with adult T-cell leukemia-lymphoma. Arq Neuropsiquiatr 58: 1107-1109, 2000.

11. Martino R, Maertens J, Bretagne S, et al. Toxoplasmosis after hematopoietic stem cell transplantation. Clin Infect Dis 31: 11881195, 2000.

12. Adurthi S, Sahoo T, Chakka K, et al. Acute toxoplasmosis in nonstem cell transplant patients with haematological malignancies: a study from a regional cancer institute in South India. Hematol Oncol 26: 229-233, 2008.

13. Robak T. Hairy-cell leukemia variant: recent view on diagnosis, biology and treatment. Cancer Treat Rev 37: 3-10, 2011.

14. Ahmadzadeh A, Shahrabi $S$, Jaseb $K$, et al. BRAF mutation in hairy cell leukemia. Oncol Rev 8: 253, 2014.

15. Dasanu CA, Ichim TE, Alexandrescu DT. Inherent and iatrogenic immune defects in hairy cell leukemia: revisited. Expert Opin Drug Saf 9: 55-64, 2010.

16. Matutes E, Wotherspoon A, Brito-Babapulle V, Catovsky D. The natural history and clinico-pathological features of the variant form of hairy cell leukemia. Leukemia 15: 184-186, 2001.

17. Gerstner ER, Batchelor TT. Primary central nervous system lymphoma. Arch Neurol 67: 291-297, 2010.

18. Erdag N, Bhorade RM, Alberico RA, Yousuf N, Patel MR. Primary lymphoma of the central nervous system: typical and atypical CT and MR imaging appearances. AJR Am J Roentgenol 176: 1319-1326, 2001.

19. Utsuki S, Oka H, Abe K, et al. Primary central nervous system lymphoma in acquired immune deficiency syndrome mimicking toxoplasmosis. Brain Tumor Pathol 28: 83-87, 2011.

20. Chandana SR, Kotecha R, Al-Janadi A, Chang HT, Conley BA. Rare case of hairy cell leukemia with brain parenchymal involvement: a diagnostic dilemma. J Clin Oncol 31: e186-e188, 2013.

21. Brogden RN, Sorkin EM. Pentostatin. A review of its pharmacodynamic and pharmacokinetic properties, and therapeutic potential in lymphoproliferative disorders. Drugs 46: 652-677, 1993.

22. Shah GD, Yahalom J, Correa DD, et al. Combined immunochemotherapy with reduced whole-brain radiotherapy for newly diagnosed primary CNS lymphoma. J Clin Oncol 25: 4730-4735, 2007.

23. Cinque P, Scarpellini P, Vago L, Linde A, Lazzarin A. Diagnosis of central nervous system complications in HIV-infected patients: cerebrospinal fluid analysis by the polymerase chain reaction. AIDS 11: 1-17, 1997.

The Internal Medicine is an Open Access article distributed under the Creative Commons Attribution-NonCommercial-NoDerivatives 4.0 International License. To view the details of this license, please visit (https://creativecommons.org/licenses/ by-nc-nd/4.0/).

(C) 2016 The Japanese Society of Internal Medicine http://www.naika.or.jp/imonline/index.html 